Metab Eng. 2019 January ; 51: 1-8. doi:10.1016/j.ymben.2018.09.005.

\title{
Engineering Saccharomyces cerevisiae for production of simvastatin
}

\author{
Carly M. Bond ${ }^{\mathrm{a}}$ and Yi Tang ${ }^{\mathrm{a}, \mathrm{b}}$ \\ aDepartment of Chemical and Biomolecular Engineering, University of California, Los Angeles, \\ California 90095, United States \\ ${ }^{b}$ Department of Chemistry and Biochemistry, University of California, Los Angeles, California \\ 90095, United States
}

\begin{abstract}
Simvastatin is a semisynthetic cholesterol-lowering medication and one of the top-selling statins in the world. Currently, industrial production of simvastatin acid (SVA) is a multistep process starting from the natural product lovastatin. For this reason, there is significant interest in direct production of simvastatin from a microbial host. In this study, six heterologous biosynthetic genes were introduced into Saccharomyces cerevisiae and the acyl-donor dimethylbutyryl- $S$-methyl mercaptopropionate (DMB-SMMP) was added, resulting in initial production of $0.5 \mathrm{mg} / \mathrm{L} \mathrm{SVA}$. Switching the yeast strain from JHY686 to BJ5464-NpgA increased total polyketide production to over $60 \mathrm{mg} / \mathrm{L}$ and conversion from dihydromonacolin $\mathrm{L}$ acid to monacolin $\mathrm{J}$ acid (MJA) was increased from 60 to $90 \%$ by tuning the copy number of the $\mathrm{P} 450$ lov $A$. Increasing the media $\mathrm{pH}$ to 8.7 led to a further 10-fold increase in SVA production. Optimized chemical lysis of the cell walls in situ after maximum MJA production led to $55 \mathrm{mg} / \mathrm{L}$ SVA titer, representing nearly complete conversion from MJA and a 110-fold increase in titer from the initial SVA production strain. The yeast strains developed in this work can be used as an alternative production method for SVA, and the strategies employed can be broadly applied for heterologous production of other fungal polyketides and semisynthetic compounds in yeast.
\end{abstract}

\section{Graphical abstract}

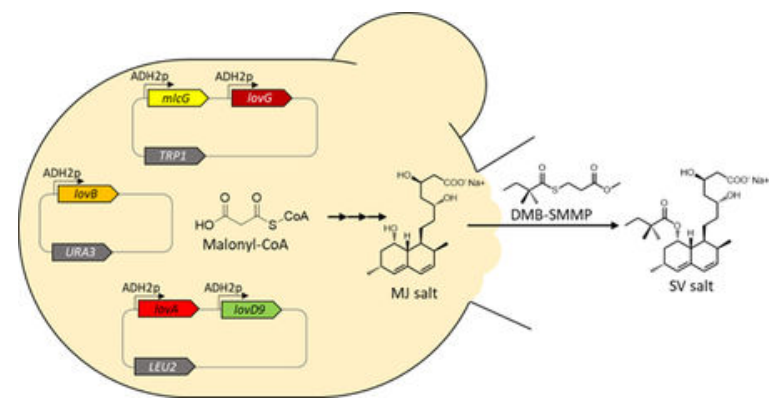

Publisher's Disclaimer: This is a PDF file of an unedited manuscript that has been accepted for publication. As a service to our customers we are providing this early version of the manuscript. The manuscript will undergo copyediting, typesetting, and review of the resulting proof before it is published in its final citable form. Please note that during the production process errors may be discovered which could affect the content, and all legal disclaimers that apply to the journal pertain. 


\section{Keywords}

Simvastatin; natural products; polyketides; Saccharomyces cerevisiae

\section{Introduction}

Small molecule secondary metabolites produced by plants, bacteria, and fungi have been an abundant source of pharmaceuticals; nearly half of the drugs approved by the U.S. Food and Drug Administration (FDA) from 1994-2008 were derived from natural products.

Polyketides are one of the major classes of natural products, and fungi in particular have been important sources of polyketide pharmaceuticals. The diverse biological activities of polyketides originate from their structural complexity, but this same complexity poses challenges for developing cost-effective methods of chemical synthesis. For this reason, fermentation of the native producing strains, followed by semi-synthesis when necessary, is used to produce nearly all commercial polyketide-derived pharmaceuticals'. The natural product lovastatin is a polyketide that was first isolated from the filamentous fungus Aspergillus terreus in 1979 and has since become a major drug used to treat hypercholesterolemia. Simvastatin is a semisynthetic compound derived from lovastatin, with a single methyl group addition on the side chain, and is one of the most highly prescribed cholesterol lowering medications, with annual sales topping $\$ 5$ billion before generics were permitted in 2007, and it is listed as an essential drug by the World Health Organization".

The lovastatin pathway has been well-studied through genetic and biochemical characterizations (Fig. 1). LovB, a highly reducing polyketide synthase (HR-PKS), and LovC, an enoyl reductase, together synthesize dihydromonacolin L acid (DMLA) from one acetyl-CoA and eight malonyl-CoA units, along with a methyl donor, $S$-adenosyl methionine (SAM), and the cofactor, nicotinamide adenine dinucleotide phosphate (NADPH). DMLA is then released from LovB by the thioesterase LovG and converted to monacolin L acid (MLA) and then monacolin J acid (MJA) by the cytochrome P450 monooxygenase (P450) LovA'. During the multistep oxidation reactions, LovA receives reducing equivalents from its redox partner, the cytochrome $\mathrm{P} 450$ reductase (CPR). The diketide synthase LovF synthesizes the a-S-methylbutyryl-ACP product, and the acyl unit is transferred to MJA by the acyltransferase LovD, completing the synthesis of lovastatin acid (LVA)'.

The semisynthetic analog simvastatin, with an a,a-dimethylbutyryl side chain, is a more effective cholesterol lowering drug and is the active pharmaceutical ingredient of the blockbuster drug Zocor ${ }^{\circledR}$ ". The semisynthesis of simvastatin from lovastatin is a multistep chemical processes that include protection and deprotection steps, requiring multiple purifications steps and is of poor atom economy"'. Our lab developed a biocatalytic process to shorten the chemical semisynthetic steps. We identified that LovD is a promiscuous acyltransferase and can directly transfer a small molecule acyl donor, a-dimethylbutyryl-Smethyl-mercaptopropionate (DMB-SMMP), to MJA to yield SVA. Further protein engineering efforts led to a LovD variant, LovD9, that has an 1000-fold increase in activity towards DMB-SMMP in the synthesis of SVA from MJA'. Although this biocatalytic 
process is used industrially to produce SVA, it still requires separate fermentation of $A$. terreus to produce lovastatin, followed by chemical hydrolysis to yield MJA and the use of LovD9 produced from Escherichia coli. Therefore, there remains significant interests in developing a one-step fermentation process for producing simvastatin from a single organism.

The native lovastatin host, $A$. terreus, has proven resistant to engineering attempts to produce simvastatin directly in our lab and in others6. E. coli, while successfully used to express LovD9, cannot express functional forms of the polyketide synthase LovB. A coculture of MJA-producing A. terreus and LovD9-expressing E. coli would require nontrivial engineering to ensure stable growth and maintenance of both organisms. Though cellfree approaches avoid the challenges of balancing organism growth and uptake of exogenously added substrates, current methods of preparing fungal extracts for cell-free synthesis are expensive and often limited by low levels of protein expression (especially with large PKS enzymes and endosome-bound P450s)". Refactoring the pathway to MJA in model yeast organisms is therefore an attractive approach. Recently, lovastatin was produced in Pichia pastoris coculture, which required separate preparations of the two P. pastoris strains expressing the upstream DMLA synthesis pathway or the downstream pathway to convert DMLA to LVA. In addition to requiring balancing the growth of these two strains, the limited ability of MJA and DMB-SMMP to cross the P. pastoris cell membrane could prove challenging for SVA production. Therefore, we focused on producing simvastatin in Saccharomyces cerevisiae. From our previous work in identifying the function of LovG, $S$. cerevisiae was used as a heterologous host to produce $20 \mathrm{mg} / \mathrm{L}$ MJA via expression of LovG, LovC, LovB, A. terreus CPR, and LovA from three separate $2 \mu$ plasmids 11 . Here, we aimed to improve MJA production in $S$. cerevisiae through refactoring of the native $A$. terreus MJA pathway and to develop a one-pot process in which SVA can be produced (Fig. $1)$.

\section{Materials and Methods}

\subsection{Strains and medium}

Saccharomyces cerevisiae strains descended from BJ5464- NpgA and BY4741 were compared as biosynthetic hosts'. Strains and modifications are summarized in Table 1. Engineered yeast strains were grown either in SD medium lacking leucine, tryptophan, histidine, and/or uracil where applicable, or in YPD medium (2\% dextrose), supplemented with $0.2 \mathrm{~g} / \mathrm{L}$ hygromycin or G418 when applicable. E. coli strains were used for cloning and followed standard recombinant DNA techniques.

\subsection{Plasmid and strain construction}

DNA restriction enzymes were acquired from New England Biolabs, NEB. Q5® HighFidelity DNA Polymerase (NEB) and AccuPrime ${ }^{\mathrm{TM}}$ Pfx DNA Polymerase (Invitrogen ${ }^{\mathrm{TM}}$ ) were used for PCR.

Gene knockouts and integrations were accomplished via CRISPR-Cas9, following the protocol outlined in mode iv from Horwitz et al. pCRCT was a gift from Huimin Zhao 
(Addgene plasmid \# 60,621) bearing iCas9. The plasmids bearing gRNAs encoded antibiotic markers KanMX and HygR. For integrations and knockouts in the BJ5464-descended strains, the plasmid was transformed as three linear pieces: One piece with the iCas9 cassette and two with the plasmid backbone and gRNA cassette that used the gRNA target sequence as a homology arm. The gRNA target sequences were designed using E-CRISP and CRISPRdirect. Genome sequences were retrieved from the Saccharomyces Genome Database (SGD). The gRNA cassettes were ordered as gBlocks from IDT. Oligonucleotides were also ordered from IDT. Plasmids assembled via yeast homologous recombination (YHR) in the course of the first knockout at some loci were purified and sequenced. These assembled plasmids were used for subsequent modifications at the same loci in other strains and are listed in Table S5.

Metabolite production plasmids are listed in Table 2. Plasmids were assembled via yeast homologous recombination, restriction-digestion cloning, or SLIC. Primers are listed in Table S1. Gene sequences and sources are listed in Table S2. Gblock sequences are listed in Table S3. Integration loci and gRNA target sequences are listed in Table S4. Additional plasmids used during construction are listed in Table S5.

\subsection{Yeast cultivation and lysis}

Transformation of $S$. cerevisiae strains was accomplished by the standard lithium acetate method. Transformants were selected on plates of YPD with $0.2 \mathrm{~g} / \mathrm{L}$ hygromycin or G418, or on uracil, leucine, histidine and/or tryptophan dropout SD medium. Colonies of JHY686 and descendants were visible 48 hours after plating while colonies of YRC01 and descendants required 72 hours. Single colony transformants were restreaked onto YPEG or onto SD dropout media if plasmid-bearing. Starter cultures were prepared by inoculating $2-$ $3 \mathrm{~mL}$ of selective media with a portion of the yeast restreak. This starter culture was shaken at $28{ }^{\circ} \mathrm{C}$ and $250 \mathrm{rpm}$ for $24-48 \mathrm{~h}$. Next, the appropriate volume of starter culture was spun down and resuspended in $9 \mathrm{~mL}$ of YPD to reach OD600 $=0.1$. This new culture was aliquoted to 3 culture tubes, $3 \mathrm{~mL}$ in each, for each condition to be tested. The cultures were shaken at $28^{\circ} \mathrm{C}$ and $250 \mathrm{rpm}$ for 72 hours until lysis, unless otherwise noted.

For glass bead mechanical lysis, $1.5 \mathrm{~mL}$ of $0.5 \mathrm{~mm}$ diameter glass beads from Biospec Products were added to the three $\mathrm{mL}$ culture, along with $250 \mu \mathrm{L}$ of $1 \mathrm{M}$ Tris- $\mathrm{HCl}$ buffer $\mathrm{pH}=8.7$. The culture was then vortexed for 30 seconds three times, resting on ice for $1-5 \mathrm{~min}$ between vortexing rounds. For chemical lysis, $250 \mu \mathrm{L}$ of Y-PER ${ }^{\mathrm{TM}}$ Yeast Protein Extraction Reagent from ThermoFisher Scientific or the described amounts of 3-(N,Ndimethylmyristylammonio)propanesulfonate (SB3-14) from Sigma-Aldrich were added to the culture tube, along with $250 \mu \mathrm{L}$ of $1 \mathrm{M}$ Tris- $\mathrm{HCl}$ buffer $\mathrm{pH}=8.7$. After lysis, 4 or $16 \mu \mathrm{L}$ $0.2 \mathrm{~g} / \mathrm{mL}$ DMB-SMMP in DMSO was added to each culture. DMB-SMMP was synthesized as previously described. Finally, cultures were shaken at $28{ }^{\circ} \mathrm{C}$ and $250 \mathrm{rpm}$. Directly before lysis and 24 hours post-lysis $100 \mathrm{uL}$ samples were taken, unless otherwise noted. In the case of YCB09, $1 \mu \mathrm{L}$ of $0.2 \mathrm{~g} / \mathrm{mL}$ DMB-SMMP in DMSO was added at 24, 36, 48, and 60 hours post inoculation into YPD and samples were taken 72 hours post inoculation. 


\subsection{Culture extraction and quantification}

In a $1.5 \mathrm{~mL}$ microcentrifuge tube, $100 \mu \mathrm{L}$ culture and $\sim 40 \mu \mathrm{L} 0.5 \mathrm{~mm}$ glass beads were combined. This was extracted twice with an organic phase of $200 \mu \mathrm{L} 2 \%$ trifluroacetic acid (TFA) in ethyl acetate (EtOAc). Samples were vortexed for 30 seconds and centrifuged for 5 minutes before the organic phase was removed. After extraction, the organic phase was dried in a refrigerated CentriVap Concentrator from LabConco for 1 hour. Samples were resuspended in four times the original sample volume of $0.1 \mathrm{M} \mathrm{NaOH}$ in methanol $(\mathrm{MeOH})$. For separate cell and media extraction, the cell pellet was spun down and the supernatant aspirated and stored in a separate tube. The two fractions were separately extracted following the above protocol. Chromatography analyses were performed on a Shimadzu 2020 EVLC-MS (Phenomenex kinetex, $1.7 \mu \mathrm{m}, 2.0 \times 100 \mathrm{~mm}$, C18 column) using positive and negative mode electrospray ionization with a linear gradient of 5-95\% acetonitrile $(\mathrm{MeCN})-\mathrm{H} 2 \mathrm{O}$ supplemented with $0.1 \%$ (v/v) formic acid in $15 \mathrm{~min}$ followed by $95 \%$ $\mathrm{MeCN}$ for $5 \mathrm{~min}$ with a flow rate of $0.3 \mathrm{~mL} / \mathrm{min}$. Standard curves were produced using DMLA, MJA, and SVA. The DMLA standard curve was used to quantify MLA by assuming an equivalent mass response factor. All measurements were taken in biological triplicate unless otherwise noted.

\subsection{Growth rate studies}

A portion of cells from a single-colony restreak of the appropriate culture was inoculated into 2-3 mL starting culture YPD. This starter culture was shaken at $28^{\circ} \mathrm{C}$ and $250 \mathrm{rpm}$ for $24 \mathrm{~h}$. Next, $100 \mu \mathrm{L}$ of YPD was inoculated to $\mathrm{OD}_{600}=0.01$ in a Corning96fc UV transparent plate with the applicable conditions. The plate was covered with Microseal ${ }^{\circledR}$ 'B' PCR Plate Sealing Film, adhesive, optical \#msb1001 from BioRad. Growth curves were measured on a Tecan Infinite M200 Pro plate reader. The program temperature was set for $29.0^{\circ} \mathrm{C}$, with $28.5^{\circ} \mathrm{C}$ minimum and $30.0^{\circ} \mathrm{C}$ maximum. The program was run for 99 cycles of 120 seconds of linear $5 \mathrm{~mm}$ shaking ( $330.5 \mathrm{rpm}$ ), absorbance was measured at $600 \mathrm{~nm}$, then 1000 seconds of orbital $3 \mathrm{~mm}$ shaking (218.3 rpm). Conditions were tested in biological triplicate, and the means were reported.

\subsection{Plasmid copy number measurements}

Colony forming units (CFU) assays were performed by removing $5 \mu \mathrm{L}$ samples of culture, diluting the cultures 20,000 -fold in sterile water, and plating $5 \mu \mathrm{L}$ on $60 \mathrm{~mm}$ diameter plates containing either YPAD or selective minimal media with uracil, leucine, or tryptophan dropouts. YPAD plates were done in technical duplicate and dropout plates were done in technical singlet. Each time point was done in biological triplicate.

Total DNA extraction was performed using the method from Lõoke et al. The volume of culture sample taken was equivalent to one $\mathrm{OD}_{600}$ unit. $\mathrm{An} \mathrm{OD}_{600}$ unit of 1 is the number of cells dissolved in $1 \mathrm{~mL}$ that gives $\mathrm{OD}_{600}=1$. Samples were spun down for 30 seconds at $15,000 \mathrm{rpm}$ and supernatant was aspirated. The resulting pellet was frozen at $-20^{\circ} \mathrm{C}$. Later the pellet was resuspended in $100 \mu \mathrm{L}$ water and $10 \mu \mathrm{L}$ of the resuspension was used for the extraction. The concentration of the resulting extracted DNA was measured and $2 \mathrm{ng}$ was used per qPCR reaction, performed using Luna ${ }^{\circledR}$ Universal qPCR Master Mix from NEB on a Bio-Rad CFX96 ${ }^{\mathrm{TM}}$ Real-Time System and C1000 ${ }^{\mathrm{TM}}$ Thermal Cycler. Results were 
quantified using the standard curve method. The reference gene used was alg9. The targeted genes representing the three plasmids were $\operatorname{lov} B, \operatorname{lov} G$, and $\operatorname{lov} D 9$. The primers used for qPCR are in Table S1.

\section{Results and Discussions}

\subsection{MJA pathway construction and production of SVA in S. cerevisiae BY4741 series}

To construct an SVA production strain, we split our proposed pathway into three modules as shown in Fig. 1. Module 1 involves the synthesis of the first dedicated precursor, DMLA, and requires the expression of LovB, LovG, and LovC. Module 2 involves the conversion of DMLA to MLA and MJA and requires the expression of LovA and CPR. The final module requires expression of LovD9 and exogenous addition of DMB-SMMP to afford SVA. The $\mathrm{ADH} 2$ promoter and terminator were chosen for all the gene cassettes. ADH2p is a strong promoter that is repressed in the presence of glucose and induced in the presence of ethanol. Reports from different groups have described that use of $\mathrm{ADH} 2 \mathrm{p}$ for episomal expression leads to greater and more sustained expression, and can even allow successful production from plasmid-based genes through fermentation in complex media". The initial $S$. cerevisiae strain we tested was JHY686, which is derived from the BY4714 series, and has repaired petite tendency, increased respiration growth rate, required protease knockouts for PKS expression, and integration of the phosphopantetheinyl transferase gene $n p g A$ to enable posttranslational modification of the expressed PKSs. To accommodate rapid genome edits in this strain, we integrated a single copy of iCas9 in the YPRCTy1-2 locus using leu2marker mediated integration, forming YCB03. Subsequent integrations and knockouts were then accomplished via transformation of the gRNA cassette and the plasmid backbone as two separate pieces, as discussed in mode iv of Horwitz et al. For sequential genome modifications, we alternated the marker on the gRNA backbone between one conferring resistance to hygromycin and another conferring resistance to G418. This allowed for rapid sequential genome edits without curing plasmids in between steps.

LovB catalyzes over thirty separate reactions in the synthesis of DMLA, so synthesis of this first dedicated precursor was anticipated to be the limiting step. Therefore, we decided to express Module 1 on high copy plasmids. In order to optimize DMLA production, we compared lovC with a related homologue. The mevastatin (also called compactin) cluster of Penicillium citrinum has been found to contain many genes with significant homology and identical putative functions to those in the lovastatin pathway'. The enoyl reductase MlcG, homologue of LovC in P. citrinum, has been shown to be functionally equivalent to LovC in the lovastatin pathway when combined with LovB in vitro. DMLA production in $S$. cerevisiae carrying plasmids encoding $\operatorname{lov} B, \operatorname{lov} G$, and $m l c G$ instead of the $A$. terreus native lovCled to $50 \%$ increase in DMLA titer (Fig. S1), therefore we chose to express $m l c G$ in Module 1. YCB03 was transformed with the Module 1 plasmids pLovB (lovB) and pCB27 ( $m / c G$, lovG), leading to an initial DMLA titer of $10 \mathrm{mg} / \mathrm{L}$ (Fig. 2A).

The next step was to incorporate Module 2, $\operatorname{lov} A$ and $C P R$, into the DMLA-producing strain to afford conversion to MLA and MJA. As is common amongst P450s, LovA is targeted to the endoplasmic reticulum (ER)'. Recently, Trenchard et al showed that expression of an ERtargeted P450 from a high copy plasmid can cause ER membrane proliferation stress and 
lower production of the desired heterologous product. To avoid this, Module 2 was integrated into the genome of the production strains. The $A$. terreus $C P R$ was integrated at the ura3 locus in YCB03, forming YCB05. Knocking out $p y c 2$ (involved in reverse glycolysis) or nte1 (involved in lipid biosynthesis) has been shown to increase the acetylCoA pool in yeast. As acetyl-CoA is a precursor to malonyl-CoA, these mutations could increase production of DMLA as well. Therefore, nte1 and pyc2 in YCB05 were knocked out sequentially and replaced by the lovA expression cassette, forming YCB06 and YCB07 respectively. After transformation with the Module 1 plasmids (pLovB and pCB27), total polyketide titer in YCB06 and YCB07 increased to $20 \mathrm{mg} / \mathrm{L}$ and $25 \mathrm{mg} / \mathrm{L}$, respectively.

Despite doubling the total polyketide titer, conversion from DMLA to MJA was only 5-10\% (Fig. 2A). For the next step, we hypothesized that further converting MJA to SVA would increase the flux of DMLA to MJA. To test this, we integrated a copy of lovD9, the evolved version of the acyltransferase lovD, in the HO locus of YCB07, forming YCB09. YCB09 transformed with pLovB and pCB27 was cultured in YPD and DMB-SMMP was added exogenously at $24,36,48$, and 60 hours post inoculation to a final concentration of 1.22 $\mathrm{mM}$. While expressing LovD9 increased the percent conversion of DMLA to MJA to 44\%, the final SVA titer was only $0.5 \mathrm{mg} / \mathrm{L}$, indicating that conversion from MJA to SVA was the limiting step, and the total polyketide production decreased to $20 \mathrm{mg} / \mathrm{L}$ (Fig. 2A, B). Based on these initial results, we aimed to increase the total amount of polyketide produced, followed by improving conversion through the pathway to MJA and SVA.

\subsection{Optimization of Total Polyketide Production and Conversion to MJA}

Due to the low initial production of polyketide in YCB03, production of DMLA was explored in YRC01, a descendent of BJ5464-NpgA with the A. terreus CPR integrated in the genome at the ura3 locus. Comparison of these strains transformed with pLovB and pCB21 showed that YRC01 produced twice as much polyketide as YCB03, justifying a shift in base strain (Fig. S2). JHY686 has a doubling time of 1.6 hours compared to the 2.4-hour doubling time of YRC01, so the lower titer in polyketide production could be due to JHY686descended strains having higher carbon flux towards growth than polyketide production. CRISPR-Cas9 was used for gene knockouts and integrations in YRC01. In order to avoid any marker-mediated integrations and to reduce any effects genomic expression of iCas9 had on polyketide production, we expressed iCas 9 and the gRNA cassette together on the same plasmid. In addition to the donor DNA with appropriate homology for the targeted locus, strains targeted for genome edits were transformed with three linear pieces of DNA that were assembled by homologous recombination in vivo into a single plasmid expressing the iCas 9 cassette and gRNA cassette for targeting the desired locus.

Using this system, copies of $\operatorname{lov} A$ were integrated into either the $\mathrm{X}-2$ or the $p y c 2$ locus to yield YCB11 and YCB19, respectively. CRISPR-Cas9 mediated integrations in YRC01 proved to be less efficient than in JHY686, potentially due to sequence differences between the genome of YRC01 and the reference yeast genome from SGD. After transforming pLovB and pCB21, the polyketide titers from YRC01, YCB11 and YCB19 were measured to be $65 \mathrm{mg} / \mathrm{L}, 30 \mathrm{mg} / \mathrm{L}$ and $42 \mathrm{mg} / \mathrm{L}$, respectively (Fig. 3A). Together, these results indicate that the genome expression of $\operatorname{lov} A$ decreases production but the $p y c 2$ knockout can improve 
total polyketide production. With only one integrated copy of $\operatorname{lov} A$, the percent conversion from DMLA to MJA was $60-65 \%$, indicating that more copies of lovA may be necessary for sufficient production of MJA.

To increase the amount of LovA expressed in the different hosts, we transformed a third plasmid, pCB19 (lovD 9 and lovA), into YRC01, YCB11, and YCB19 along with the Module 1 plasmids. This significantly improved conversion of DMLA to MJA as 85\%-90\% conversion was observed in all three strains (Fig. 3A). The total polyketide titer, however, varied between the strains. For YRC01, both genome (as in YCB11) and episomal expression (with pCB19) of LovA led to decreases in total titer from $66 \mathrm{mg} / \mathrm{L}$ to 31 and 30 $\mathrm{mg} / \mathrm{L}$, respectively. This indicates the decrease in yield is a result of introducing the P450 into the yeast host, regardless of copy number of the gene. However, addition of pCB19 to YCB11 and YCB19, which both already expressed single integrated copies of $\operatorname{lov} A$, did not further decrease the total polyketide titer. Notably, YCB19 transformed with all three plasmids had the highest polyketide production with $90 \%$ conversion of DMLA to MJA at 72 hours post inoculation. Measurements of the polyketides produced by this strain over time indicated that MJA titer peaked at $75 \mathrm{mg} / \mathrm{L}, 72$ hours post inoculation (Fig. 3B). Contrasting the productivity of YCB11 and YCB19 further underscores the importance of the $\Delta p y c 2$ knockout for improving polyketide production. Our studies also showed that the gene dosage for P450 enzymes from biosynthetic pathways needs to be systematically tuned in $S$. cerevisiae for each pathway.

\subsection{Characterizing plasmid stability and copy numbers}

YCB19 transformed with pLovB, pCB21 and pCB19 represents the best strain to date in the production of MJA, the immediate precursor to simvastatin. The stability of the three $2 \mu$ plasmids in YCB19 was measured by determining the percentage of cells maintaining each plasmid over the 72-hours culture period. The copy numbers of the plasmids provide an initial estimate for how many integrated copies of each pathway gene would be needed to reach a similar production level.

Copy numbers of each of the plasmids in YCB19 were measured at 24, 48, and 72 hours after inoculation (Fig. 3B, Table 3). The copy number of each plasmid was similar at 24 and 72 hours after inoculation, indicating that the plasmid copy number was stable for this period of culturing. Notably, each plasmid was only carried by $\sim 40-50 \%$ of the cells at any time point, resulting in a significantly lower copy number of each plasmid when averaged over all cells. This indicates that similar levels of production and conversion to MJA could potentially be achieved by integrating less than ten copies of $\operatorname{lov} A$, rather than the twenty or more copies of pCB19 maintained by the cells. The percentage of cells carrying all three plasmids simultaneously could be even lower than $40 \%$, which would indicate that even lower integrated copy numbers of the needed genes would be sufficient to reach similar levels of production. 


\subsection{Development of an in situ lysis process to overcome substrate and enzyme localization differences}

With higher conversion to MJA achieved, the final step was to improve conversion of MJA to SVA. In the initial SVA production strain (YCB09 transformed with pLovB and pCB27), DMB-SMMP was added throughout the 72-hour culturing period and less than $1 \mathrm{mg} / \mathrm{L}$ SVA was produced. Several possible reasons were examined for this low conversion. First, growth curves of $S$. cerevisiae in the presence of DMB-SMMP indicated that it is toxic to yeast growth, which could contribute to the lower total polyketide production (Fig. S3). Second, LovD9 has optimal activity at $\mathrm{pH} 8.0$ to 9.5 , while the $\mathrm{pH}$ of $S$. cerevisiae grown in YPD is significantly lower. The intracellular $\mathrm{pH}$ inside of $S$. cerevisiae is 6-7 while the extracellular $\mathrm{pH}$ is 5-6. Third, different localizations of MJA, DMB-SMMP, and LovD9 might prevent high conversion of MJA to SVA. Finally, simvastatin and lovastatin have both been shown to greatly inhibit yeast growth at $1.3 \mathrm{mM}$ exogenous concentrations of these statins.

To circumvent DMB-SMMP toxicity, DMB-SMMP was added to YCB19 transformed with the three plasmids only at 72 hours after inoculation, when the concentration of MJA was the highest, and samples were taken 24 hours later. However, this led to only $0.5 \mathrm{mg} / \mathrm{L} \mathrm{SVA}$ (Fig. 4B). Next, Tris-HCl buffer at $\mathrm{pH} 8.7$ was added with DMB-SMMP to the cultures at 72 hours post-inoculation to improve the activity of LovD9. Increasing the culture $\mathrm{pH}$ led to a five-fold increase in SVA production to $5.9 \mathrm{mg} / \mathrm{L}$ (Fig. 4B), however, the percent conversion from MJA to SVA was still less than $15 \%$.

To further increase conversion, we investigated the localization of the produced MJA. Separate metabolite analysis of the cell pellet and the media indicated that $98 \%$ of the produced MJA has been secreted to the extracellular space (Fig. S4). As DMB-SMMP was added exogenously, a significant portion of this substrate would also be in the media. With both substrates extracellular and LovD9 expressed intracellularly, conversion to SVA was therefore expectedly low. One possible solution is to remove the cell wall barrier at the time of MJA to SVA conversion. Cell-free reactions have been used to improve production of natural products, but these systems have required spinning down cultures and resuspending in a lysis buffer or otherwise concentrating the extract, which introduces significant process steps and scale-up barriers'.

For this reason, we decided to lyse the cells in situ at 72 hours post inoculation when MJA titer was highest (Fig. 3B, Fig. 4A). Several lysis methods were tested, all performed with the increased media $\mathrm{pH}$ and addition of $1.22 \mathrm{mM}$ DMB-SMMP. Initially, the cells were lysed in situ using glass beads, however decreased conversion relative to the whole cells was observed (Fig 4B). We then tested lysis efficiency using Y-PER and the cost-effective detergent 3-(N,N-Dimethylmyristylammonio)propanesulfonate (SB3-14) as chemical lysis agents'. Y-PER and SB3-14 were both found to lead to improved SVA production, with 5 g/L SB3-14 giving the highest titer of SVA at $32 \mathrm{mg} / \mathrm{L}$ (Fig. 4B). We further determined that $2 \mathrm{~g} / \mathrm{L}$ SB3-14 was the minimum concentration required to lyse the cells completely (Fig. S5).

Using $2 \mathrm{~g} / \mathrm{L}$ of SB3-14 as the lysis agent, we monitored the conversion of MJA to SVA over time. The highest concentration of SVA occurred 16 hours after lysis (Fig. 4C,D). At longer 
time, the titer of SVA decreased, while that of MJA increased. This is expected as LovD can catalyze the reverse reaction which is the hydrolysis of SVA to MJA. Analysis of the breakdown of polyketide products formed at the time points taken indicated that the concentrations of DMLA and MLA remained low and constant throughout. Adding an increased amount of DMB-SMMP led to production of $55 \mathrm{mg} / \mathrm{L} \mathrm{SVA}$ and over $95 \%$ conversion from MJA (Fig. 4C,D). This represents the highest level of SVA produced in yeast using fermentation approaches and an 110-fold increase from the initial SVA production reached.

\section{Conclusion}

In this study, we refactored the LVA biosynthetic pathway from $A$. terreus into $S$. cerevisiae and found that higher polyketide production was reached in the BJ5464-descended strains than in the faster growing BY4741-descended strains. We found that incorporation of the P450 $\operatorname{lov} A$, whether integrated or episomally expressed, decreased total polyketide production. However, total polyketide production can be increased by knockouts of $p y c 2$ and nte 1, and could compensate for the drop in titer upon lov $A$ overexpression. High copy expression of $\operatorname{lov} A$ was necessary for the near complete conversion of DMLA to MJA. The initial SVA production from the top MJA-producing strain was $0.5 \mathrm{mg} / \mathrm{L}$, which was increased 5-fold via pH optimization and 110-fold via optimized in situ lysis, reaching 55 $\mathrm{mg} / \mathrm{L}$ SVA and nearly $100 \%$ conversion from MJA. This work marks the first time SVA has been produced using a single heterologous host, $S$. cerevisiae, and the first time in situ lysis has been used for production of a semisynthetic natural product. This provides a novel strategy that can be used by future researchers for production of semisynthetic polyketides in yeast.

\section{Supplementary Material}

Refer to Web version on PubMed Central for supplementary material.

\section{Acknowledgements}

This work was supported by National Science Foundation CBET1605877. Carly Bond was supported by the NIH T32 Biotechnology Training Grant (2 T32 GM067555). We also thank volunteers Youna Choi, Han Ngo, and Jessee Svoboda for helpful discussions and technical work. We thank Dr. Anthony DeNicola for helpful discussions regarding CRISPR-Cas9, qPCR, and growth curves and Dr. Mengbin Chen and John Billingsley for editing assistance.

\section{References}

1. Harvey AL Natural products in drug discovery. Drug Discov Today 13, 894-901, 10.1016/j.drudis. 2008.07.004 (2008). [PubMed: 18691670]

2. Hertweck C The biosynthetic logic of polyketide diversity. Angewandte Chemie 48, 4688-4716, 10.1002/anie.200806121 (2009). [PubMed: 19514004]

3. Dechert-Schmitt AM, Schmitt DC, Gao X, Itoh T \& Krische MJ Polyketide construction via hydrohydroxyalkylation and related alcohol $\mathrm{C}-\mathrm{H}$ functionalizations: reinventing the chemistry of carbonyl addition. Nat Prod Rep 31, 504-513, 10.1039/c3np70076c (2014). [PubMed: 24514754]

4. Wong FT \& Khosla C Combinatorial biosynthesis of polyketides--a perspective. Curr Opin Chem Biol 16, 117-123, 10.1016/j.cbpa.2012.01.018 (2012). [PubMed: 22342766] 
5. Alberts AW et al. Mevinolin: a highly potent competitive inhibitor of hydroxymethylglutarylcoenzyme A reductase and a cholesterol-lowering agent. Proc Natl Acad Sci U S A 77, 3957-3961 (1980). [PubMed: 6933445]

6. Barrios-Gonzalez J \& Miranda RU Biotechnological production and applications of statins. Appl Microbiol Biotechnol 85, 869-883, 10.1007/s00253-009-2239-6 (2010). [PubMed: 19820926]

7. Lamon-Fava S Statins and lipid metabolism: an update. Curr Opin Lipidol 24, 221-226, 10.1097/ MOL.0b013e3283613b8b (2013). [PubMed: 23571733]

8. Weng TC, Yang YH, Lin SJ \& Tai SH A systematic review and meta-analysis on the therapeutic equivalence of statins. J Clin Pharm Ther 35, 139-151, 10.1111/j.1365-2710.2009.01085.x (2010). [PubMed: 20456733]

9. Kennedy $\mathrm{J}$ et al. Modulation of polyketide synthase activity by accessory proteins during lovastatin biosynthesis. Science (New York, N.Y.) 284, 1368-1372 (1999).

10. Wagschal K, Yoshizawa Y, Witter DJ, Liu YQ \& Vederas JC Biosynthesis of ML-236C and the hypocholesterolemic agents compactin by Penicillium aurantiogriseum and lovastatin by Aspergillus terreus: Determination of the origin of carbon, hydrogen and oxygen atoms by C-13 NMR spectrometry and observation of unusual labelling of acetate-derived oxygens by O-18(2). J Chem Soc Perk T 1, 2357-2363, doi:Doi 10.1039/P19960002357 (1996).

11. $\mathrm{Xu} \mathrm{W}$ et al. LovG: the thioesterase required for dihydromonacolin $\mathrm{L}$ release and lovastatin nonaketide synthase turnover in lovastatin biosynthesis. Angewandte Chemie 52, 6472-6475, 10.1002/anie.201302406 (2013). [PubMed: 23653178]

12. Barriuso $\mathrm{J}$ et al. Double oxidation of the cyclic nonaketide dihydromonacolin $\mathrm{L}$ to monacolin $\mathrm{J}$ by a single cytochrome P450 monooxygenase, LovA. J Am Chem Soc 133, 8078-8081, 10.1021/ ja201138v (2011). [PubMed: 21495633]

13. Xie X, Meehan MJ, Xu W, Dorrestein PC \& Tang Y Acyltransferase mediated polyketide release from a fungal megasynthase. J Am Chem Soc 131, 8388-8389, 10.1021/ja903203g (2009). [PubMed: 19530726]

14. Hoffman WF et al. 3-Hydroxy-3-methylglutaryl-coenzyme A reductase inhibitors. 4. Side chain ester derivatives of mevinolin. Journal of medicinal chemistry 29, 849-852 (1986). [PubMed: 3634830]

15. Tobert JA Lovastatin and beyond: the history of the HMG-CoA reductase inhibitors. Nature reviews. Drug discovery 2, 517-526, 10.1038/nrd1112 (2003). [PubMed: 12815379]

16. Freeman MW Statins, cholesterol, and the prevention of coronary heart disease. Faseb J 20, 200 201, 10.1096/fj.06-0202ufm (2006). [PubMed: 16449790]

17. Berg VDMA, Hans M \& Streekstra H Method for the production of simvastatin WO2007147801A1 (2007).

18. Morgan B et al. Methods for making simvastatin and intermediates WO2005040107A2 (2005).

19. Askin D, Verhoeven TR, Liu TMH \& Shinkai I Synthesis of Synvinolin -Extremely High Conversion Alkylation of an Ester Enolate. Journal of Organic Chemistry 56, 4929-4932, 10.1021/ Jo00016a023 (1991).

20. Xie X, Watanabe K, Wojcicki WA, Wang CC \& Tang Y Biosynthesis of lovastatin analogs with a broadly specific acyltransferase. Chem Biol 13, 1161-1169, 10.1016/j.chembiol.2006.09.008 (2006). [PubMed: 17113998]

21. Gao X et al. Directed evolution and structural characterization of a simvastatin synthase. Chem Biol 16, 1064-1074, 10.1016/j.chembiol.2009.09.017 (2009). [PubMed: 19875080]

22. Jimenez-Oses $\mathrm{G}$ et al. The role of distant mutations and allosteric regulation on LovD active site dynamics. Nat Chem Biol 10, 431-436, 10.1038/nchembio. 1503 (2014). [PubMed: 24727900]

23. Zemella A, Thoring L, Hoffmeister C \& Kubick S Cell-Free Protein Synthesis: Pros and Cons of Prokaryotic and Eukaryotic Systems. Chembiochem : a European journal of chemical biology 16, 2420-2431, 10.1002/cbic.201500340 (2015). [PubMed: 26478227]

24. Anderson MJ, Stark JC, Hodgman CE \& Jewett MC Energizing eukaryotic cell-free protein synthesis with glucose metabolism. FEBS Lett 589, 1723-1727, 10.1016/j.febslet.2015.05.045 (2015). [PubMed: 26054976] 
25. Hodgman CE \& Jewett MC Characterizing IGR IRES-mediated translation initiation for use in yeast cell-free protein synthesis. N Biotechnol 31, 499-505, 10.1016/j.nbt.2014.07.001 (2014). [PubMed: 25017988]

26. Liu $\mathrm{Y}$ et al. Engineered monoculture and co-culture of methylotrophic yeast for de novo production of monacolin J and lovastatin from methanol. Metab Eng 45, 189-199, 10.1016/j.ymben. 2017.12.009 (2018). [PubMed: 29258964]

27. Xu W, Cai X, Jung ME \& Tang Y Analysis of intact and dissected fungal polyketide synthasenonribosomal peptide synthetase in vitro and in Saccharomyces cerevisiae. J Am Chem Soc 132, 13604-13607, 10.1021/ja107084d (2010). [PubMed: 20828130]

28. Brachmann CB et al. Designer deletion strains derived from Saccharomyces cerevisiae S288C: a useful set of strains and plasmids for PCR-mediated gene disruption and other applications. Yeast 14, 115-132, 10.1002/(SICI)1097-0061(19980130)14:2<115::AID-YEA204>3.0.CO;2-2 (1998). [PubMed: 9483801]

29. Harvey CJB et al. HEx: A heterologous expression platform for the discovery of fungal natural products. Science advances 4, eaar5459, 10.1126/sciadv.aar5459 (2018).

30. Ma SM et al. Complete reconstitution of a highly reducing iterative polyketide synthase. Science (New York, N.Y.) 326, 589-592, 10.1126/science.1175602 (2009).

31. Tang MC et al. Discovery of Unclustered Fungal Indole Diterpene Biosynthetic Pathways through Combinatorial Pathway Reassembly in Engineered Yeast. J Am Chem Soc 137, 13724-13727, 10.1021/jacs.5b06108 (2015). [PubMed: 26469304]

32. Horwitz AA et al. Efficient Multiplexed Integration of Synergistic Alleles and Metabolic Pathways in Yeasts via CRISPR-Cas. Cell systems 1, 88-96, 10.1016/j.cels.2015.02.001 (2015). [PubMed: 27135688]

33. Bao Z et al. Homology-integrated CRISPR-Cas (HI-CRISPR) system for one-step multigene disruption in Saccharomyces cerevisiae. ACS Synth Biol 4, 585-594, 10.1021/sb500255k (2015). [PubMed: 25207793]

34. Heigwer F, Kerr G \& Boutros M E-CRISP: fast CRISPR target site identification. Nature methods 11, 122-123, 10.1038/nmeth.2812 (2014). [PubMed: 24481216]

35. Naito Y, Hino K, Bono H \& Ui-Tei K CRISPRdirect: software for designing CRISPR/Cas guide RNA with reduced off-target sites. Bioinformatics (Oxford, England) 31, 1120-1123, 10.1093/ bioinformatics/btu743 (2015).

36. Engel SR et al. The reference genome sequence of Saccharomyces cerevisiae: then and now. G3 (Bethesda, Md.) 4, 389-398, 10.1534/g3.113.008995 (2014).

37. Li MZ \& Elledge SJ Harnessing homologous recombination in vitro to generate recombinant DNA via SLIC. Nature methods 4, 251-256, 10.1038/nmeth1010 (2007). [PubMed: 17293868]

38. Gietz RD \& Schiestl RH High-efficiency yeast transformation using the LiAc/SS carrier DNA/PEG method. Nat Protoc 2, 31-34, 10.1038/nprot.2007.13 (2007). [PubMed: 17401334]

39. Xie X \& Tang Y Efficient synthesis of simvastatin by use of whole-cell biocatalysis. Appl Environ Microbiol 73, 2054-2060, 10.1128/AEM.02820-06 (2007). [PubMed: 17277201]

40. Looke M, Kristjuhan K \& Kristjuhan A Extraction of genomic DNA from yeasts for PCR-based applications. BioTechniques 50, 325-328, 10.2144/000113672 (2011). [PubMed: 21548894]

41. Lee KM \& DaSilva NA Evaluation of the Saccharomyces cerevisiae ADH2 promoter for protein synthesis. Yeast 22, 431-440, 10.1002/yea.1221 (2005). [PubMed: 15849781]

42. Cardenas J \& Da Silva NA Metabolic engineering of Saccharomyces cerevisiae for the production of triacetic acid lactone. Metab Eng 25, 194-203, 10.1016/j.ymben.2014.07.008 (2014). [PubMed: 25084369]

43. Xie D et al. Microbial synthesis of triacetic acid lactone. Biotechnol Bioeng 93, 727-736, 10.1002/ bit.20759 (2006). [PubMed: 16245348]

44. Shen MW, Fang F, Sandmeyer S \& Da Silva NA Development and characterization of a vector set with regulated promoters for systematic metabolic engineering in Saccharomyces cerevisiae. Yeast 29, 495-503, 10.1002/yea.2930 (2012). [PubMed: 23166051]

45. Abe $\mathrm{Y}$ et al. Molecular cloning and characterization of an ML-236B (compactin) biosynthetic gene cluster in Penicillium citrinum. Molecular genetics and genomics : MGG 267, 636-646, 10.1007/ s00438-002-0697-y (2002). [PubMed: 12172803] 
46. Auclair K, Kennedy J, Hutchinson CR \& Vederas JC Conversion of cyclic nonaketides to lovastatin and compactin by a lovC deficient mutant of Aspergillus terreus. Bioorg Med Chem Lett 11, 1527-1531 (2001). [PubMed: 11412974]

47. Renault H, Bassard JE, Hamberger B \& Werck-Reichhart D Cytochrome P450-mediated metabolic engineering: current progress and future challenges. Current opinion in plant biology 19, 27-34, 10.1016/j.pbi.2014.03.004 (2014). [PubMed: 24709279]

48. Trenchard IJ \& Smolke CD Engineering strategies for the fermentative production of plant alkaloids in yeast. Metab Eng 30, 96-104, 10.1016/j.ymben.2015.05.001 (2015). [PubMed: 25981946]

49. Valli $\mathrm{M}$ et al. Intracellular $\mathrm{pH}$ distribution in Saccharomyces cerevisiae cell populations, analyzed by flow cytometry. Appl Environ Microbiol 71, 1515-1521, 10.1128/AEM.71.3.1515-1521.2005 (2005). [PubMed: 15746355]

50. Ley A, Coumou HC \& Frandsen RJN Heterologous expression of MlcE in Saccharomyces cerevisiae provides resistance to natural and semi-synthetic statins. Metabolic Engineering Communications 2, 117-123, 10.1016/j.meteno.2015.09.003 (2015).

51. Khattak WA et al. Developmental strategies and regulation of cell-free enzyme system for ethanol production: a molecular prospective. Appl Microbiol Biotechnol 98, 9561-9578, 10.1007/ s00253-014-6154-0 (2014). [PubMed: 25359472]

52. You C \& Zhang YH Cell-free biosystems for biomanufacturing. Advances in biochemical engineering/biotechnology 131, 89-119, 10.1007/10_2012_159 (2013). [PubMed: 23111502]

53. Yang-Boja E, DeFilippes F \& Fales HM Electrospray mass spectra of three proprietary detergents. Anal Biochem 285, 205-210, 10.1006/abio.2000.4734 (2000). [PubMed: 11017703]

54. Yamamoto E, Yamaguchi S \& Nagamune T Synergistic effects of detergents and organic solvents on protein refolding: control of aggregation and folding rates. J Biosci Bioeng 111, 10-15, 10.1016/j.jbiosc.2010.08.016 (2011). [PubMed: 20855233] 


\section{Highlights}

- $\quad$ Produced MJA and SVA heterologously in S. cerevisiae.

- Increased conversion to MJA through high copy expression of a fungal P450.

- $\quad$ Developed an in situ lysis process that increased MJA to SVA conversion 110fold.

- $\quad$ Produced $55 \mathrm{mg} / \mathrm{L}$ SVA in $S$. cerevisiae, reaching nearly complete conversion from MJA. 


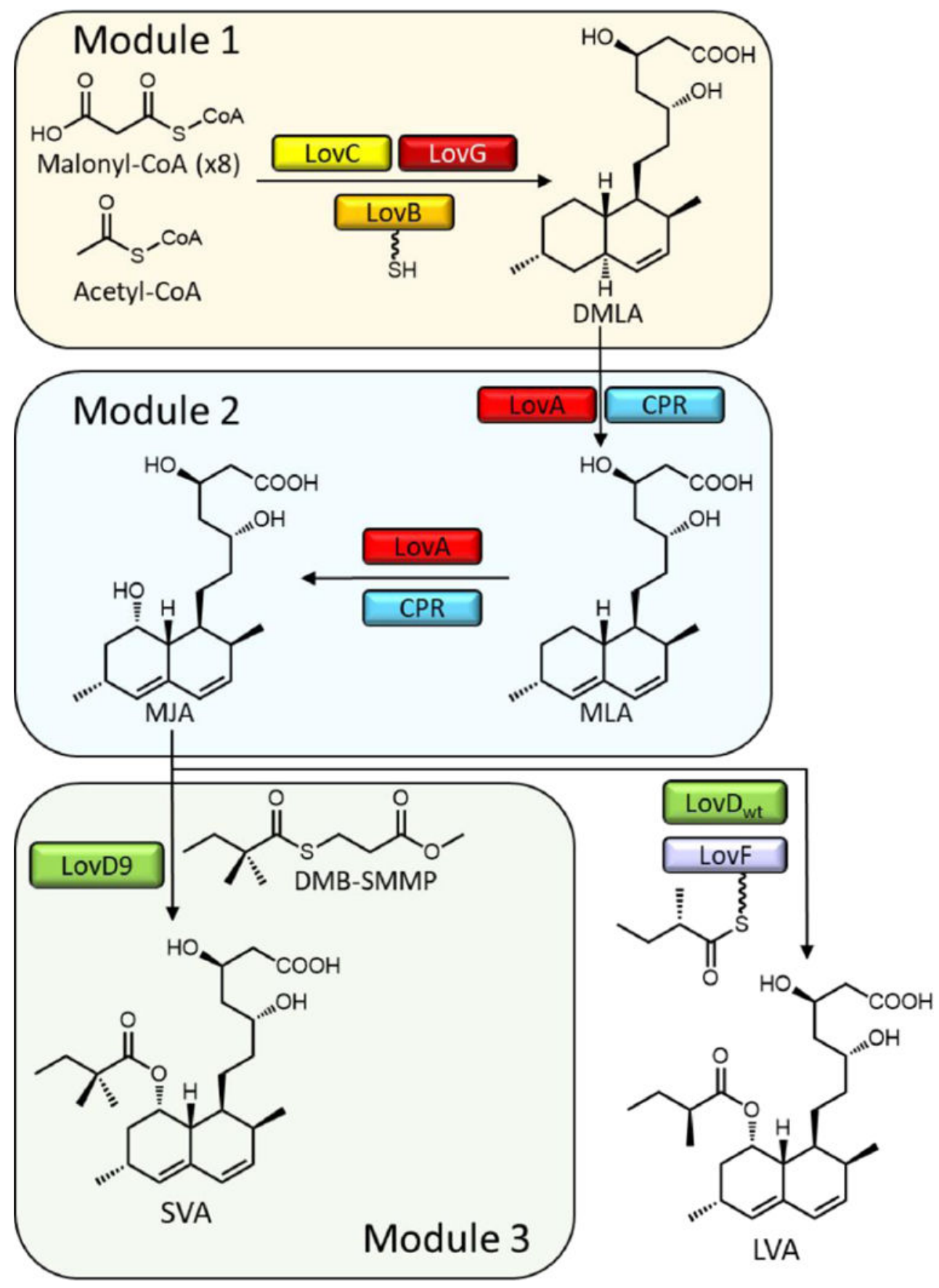

Figure 1:

Lovastatin and simvastatin biosynthesis. In Module 1, LovB (polyketide synthase) and LovC (enoyl reductase) use one acetyl-CoA and eight malonyl-CoA units to form the polyketide backbone which is released from LovB by LovG (thioesterase), forming DMLA (dihydromonacolin L acid). In Module 2, LovA (cytochrome P450 monooxygenase) catalyzes the conversion of DMLA to MLA (monacolin L acid) and then to MJA (monacolin $\mathrm{J}$ acid). LovA is reduced by its partner CPR (cytochrome P450 reductase). In Module 3, the semisynthetic acyl donor DMB-SMMP (a,a-dimethylbutyryl-S-methyl- 
mercaptoproprionate) is transferred to MJA by an evolved version of LovD (acyl transferase) to form SVA (simvastatin acid). In the native pathway, LovF (polyketide synthase) provides the acyl side chain which is transferred by the wild type LovD to form LVA (lovastatin acid). 

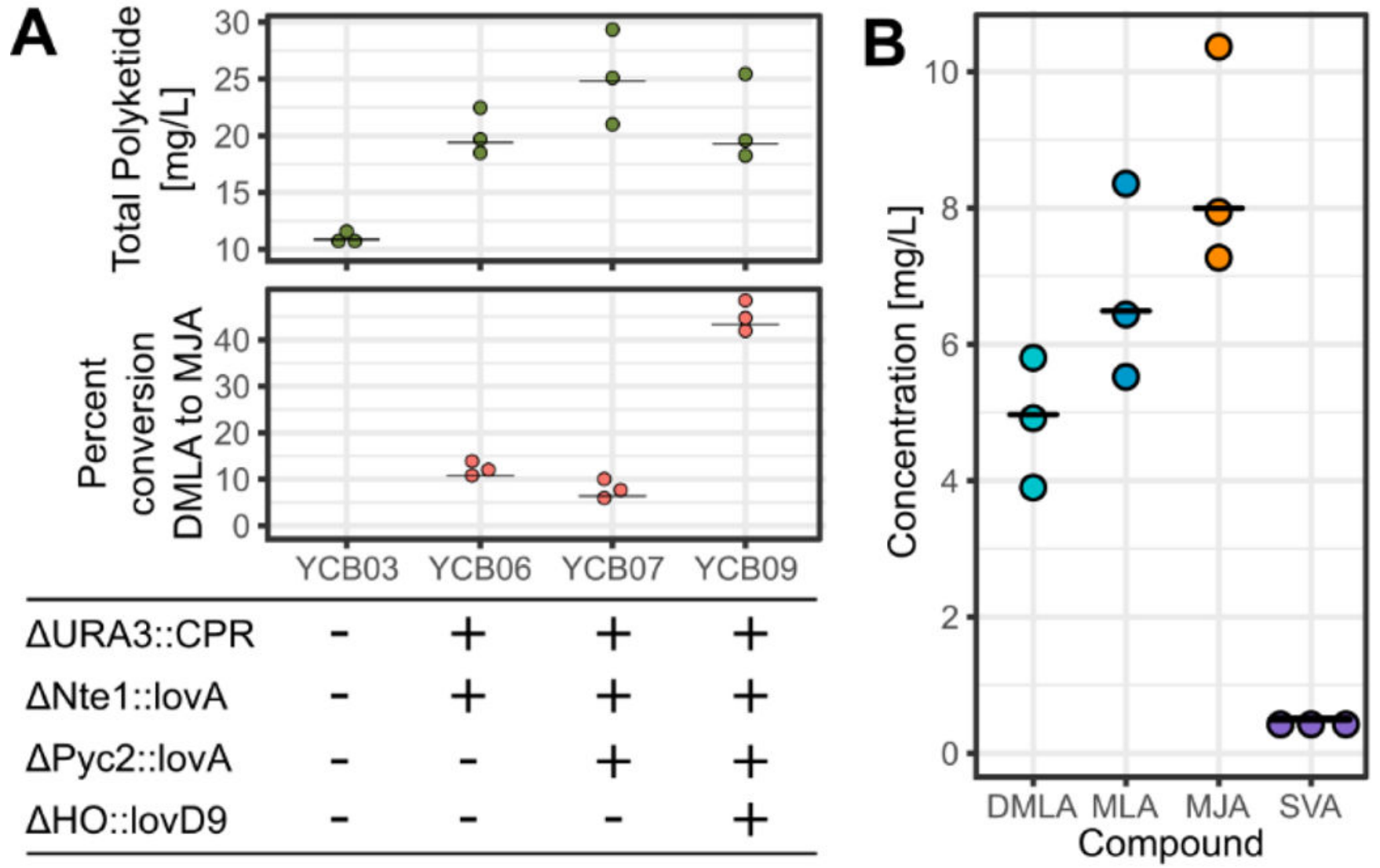

Figure 2:

Polyketide production in the BY4741-descended strains. All strains contain Module 1 plasmids pLovB and pCB27 (A) Total polyketide production in green. The percent conversion of DMLA to MJA is in orange. Each point represents measurement from a different sample. Black horizontal lines indicate median values. (B) Breakdown of polyketide production in YCB09. Results are from triplicate runs. Black horizontal lines represent the median. 

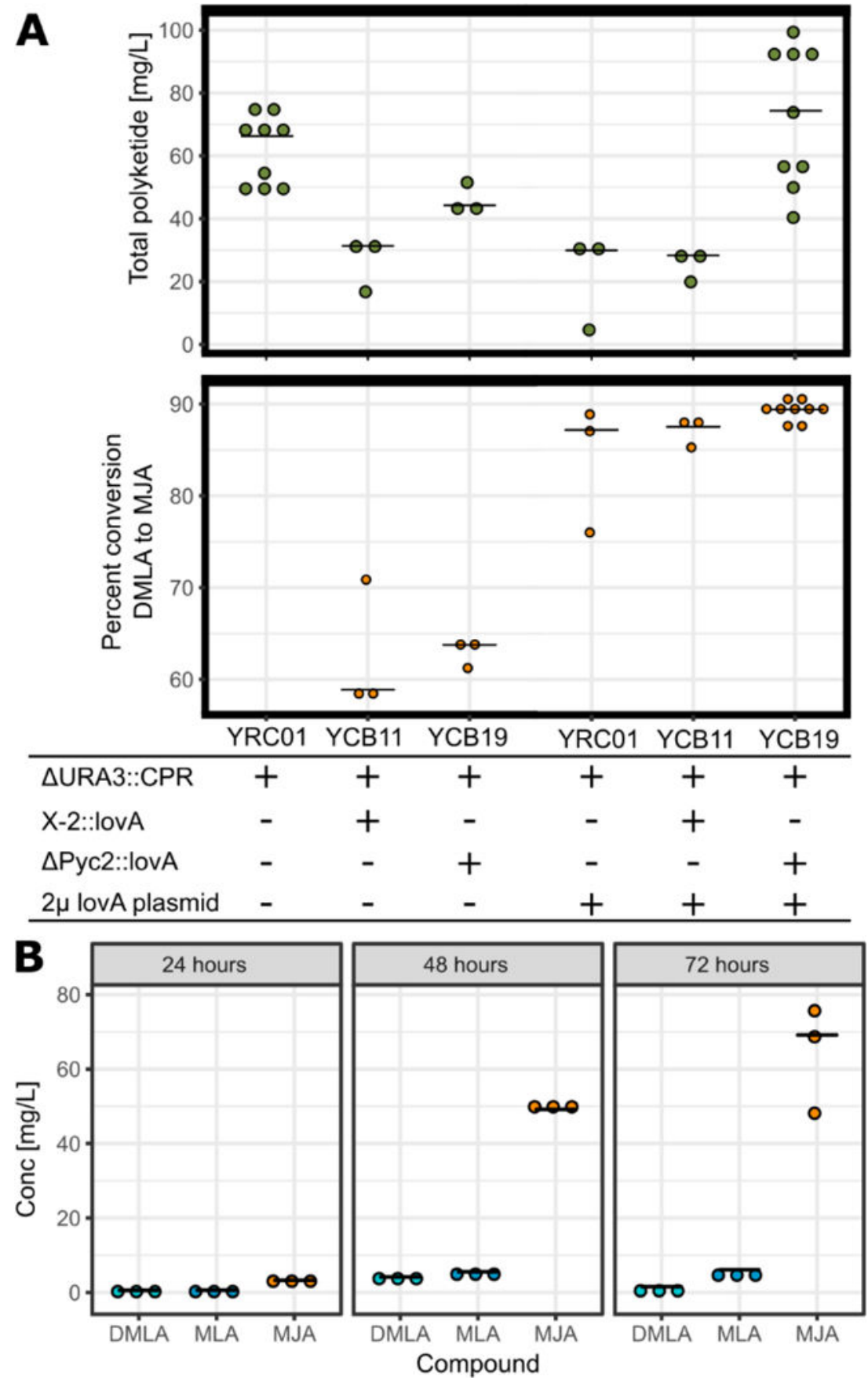

Figure 3:

Polyketide production in the BJ5464-descended strains. All strains contained the Module 1 plasmids, pLovB and pCB21. (A) Polyketide production from YRC01, YCB11, and YCB19 at 72 hours post inoculation. The final row in the table, $2 \mu$ lovA plasmid, refers to the presence or absence of pCB19. Total polyketide production in green. Percent conversion from DMLA to MJA is in orange. Each point is a different sample. Black horizontal lines indicate median values. (B) Breakdown of polyketide production in highest MJA producing 
strain, YCB19 transformed with pLovB, PCB21 and pCB19 at 24, 48, and 72 hours post inoculation into YPD. Results in triplicate. Black horizontal lines represent median values. 

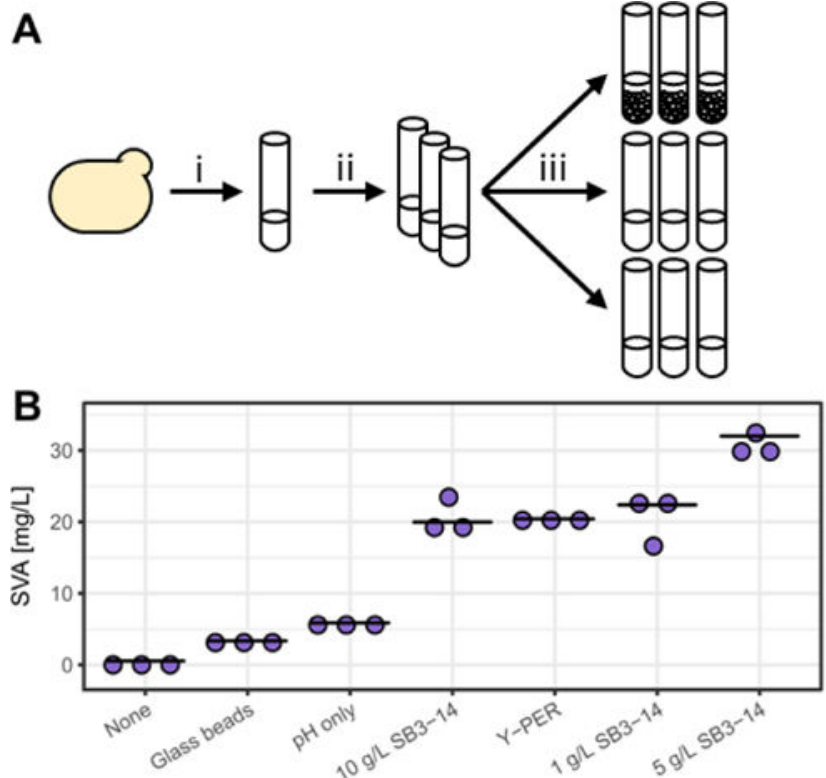
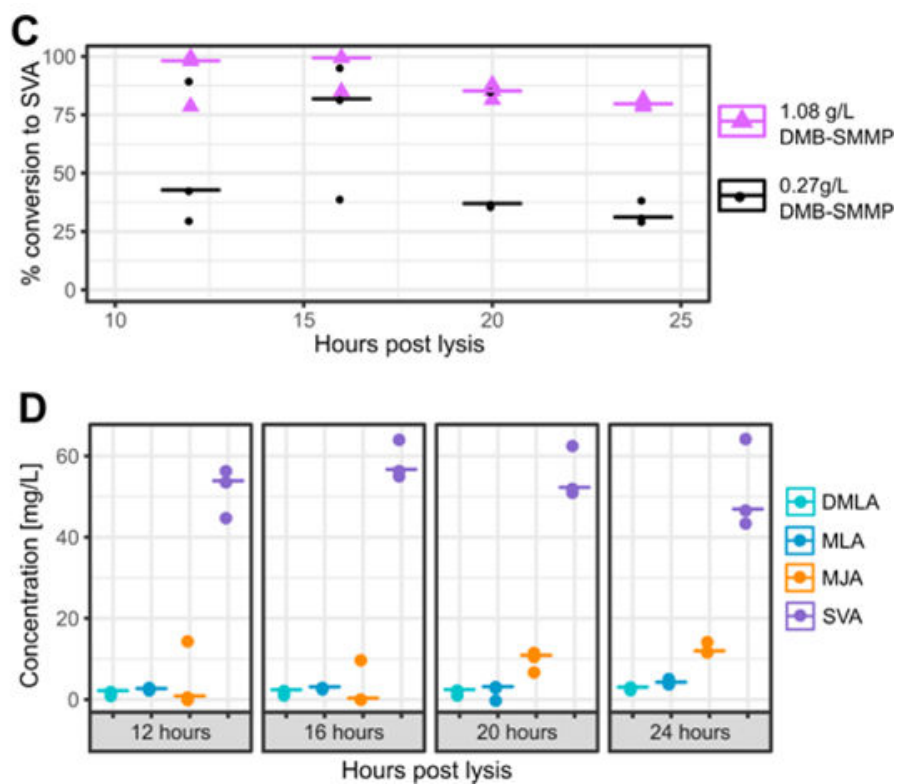

Figure 4:

SVA production from the highest MJA producing strain. (A) Schematic showing the production protocol. In step $i$, the yeast was picked into selective media. After 24 hours, triplicate cultures of YPD were inoculated from the seed in step ii. After 72 hours of culturing, the $\mathrm{pH}$ was adjusted, DMB-SMMP was added, and then the appropriate lysis method was used, as shown in step iii. Cultures were shaken for up to 24 hours more while samples were taken. (B) SVA concentration 24 hours after lysis and DMB-SMMP feeding using different lysis methods. The $\mathrm{pH}$ of all the cultures was adjusted except for the one labeled none. Each point is a different sample. The horizontal black line represents the median. (C) Time course of percent conversion of MJA to SVA after addition of $2 \mathrm{~g} / \mathrm{L}$ of SB3-14 (3-(N,N-Dimethylmyristylammonio)propanesulfonate), a chemical lysis reagent, and varied concentrations of DMB-SMMP. Results in triplicate. The horizontal lines represent the median values. (D) Breakdown of polyketide production of the highest SVA producing conditions from the time course in $(\mathrm{C})$. 
Table 1:

S. cerevisiae strains used in this work.

\begin{tabular}{|c|c|c|c|}
\hline Strain & Parent & Genotype modifications to parent & Reference \\
\hline BY4741 & S288C & 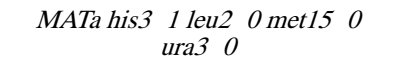 & Brachman et al, 1998 \\
\hline DHY213 & BY4741 & $\begin{array}{c}S A L 1+H A P 1+C A T 5(91 \mathrm{M}) \\
M I P 1(661 \mathrm{~T}) M K T 1(30 \mathrm{G}) \\
R M E 1(\mathrm{INS}-308 \mathrm{~A}) \operatorname{TAO3(1493\mathrm {Q})}\end{array}$ & Harvey et al, 2018 \\
\hline JHY686 & DHY213 & $\begin{array}{c}\text { prb1 } 1 \Delta \text { pep4A ADH2p-npgA- } \\
\text { ACS1t lys2AO }\end{array}$ & Harvey et al, 2018 \\
\hline YCB03 & JHY686 & YPRCTy1-2::iCas9::LEU2 & This study \\
\hline YCB06 & YRCB03 & $\begin{array}{c}\text { ura3 } 40:: A D H 2 \mathrm{p}-C P R-A D H 2 \mathrm{t} \\
\text { nte1 } 1 \Delta:: A D H 2 \mathrm{p}-l o v A-A D H 2 \mathrm{t}\end{array}$ & This study \\
\hline YCB07 & YCB06 & pyc2A::ADH2p-lovA-ADH2t & This study \\
\hline YCB09 & YCB07 & $H O:: A D H 2 \mathrm{p}-I o v A-A D H 2 t$ & This study \\
\hline $\begin{array}{l}\text { BJ5464- } \\
\text { NpgA }\end{array}$ & S288C & 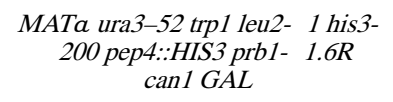 & Ma et al, 2009 \\
\hline YRC01 & $\begin{array}{l}\text { BJ5464- } \\
\text { NpgA }\end{array}$ & ura3A: $A D H 2 \mathrm{p}-C P R-A D H 2 \mathrm{t}$ & Tang et al, 2015 \\
\hline YCB11 & YRC01 & $X-2:: A D H 2 \mathrm{p}-\operatorname{lov} A-A D H 2 \mathrm{t}$ & This study \\
\hline YCB19 & YRC01 & pyc2A::ADH2p-lovA-ADH2t & This study \\
\hline
\end{tabular}

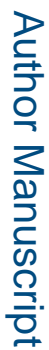

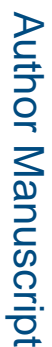

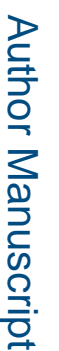


Table 2:

Production plasmids used in this work

\begin{tabular}{|c|c|c|}
\hline $\begin{array}{l}\text { Production } \\
\text { Plasmid }\end{array}$ & Description & Reference \\
\hline pLovB & $\begin{array}{l}2 \mu ; A m p R ; U R A 3 ; A D H 2 \mathrm{p}-1 o v B-6 \mathrm{xHis}- \\
A D H 2 \mathrm{t}\end{array}$ & YEpLovB-6His from Xu, et al, 2013 \\
\hline pGC & $\begin{array}{l}2 \mu ; A m p R ; T R P 1 ; A D H 2 \mathrm{p}-l o v C-6 \mathrm{xHis}- \\
A D H 2 t ; A D H 2 \mathrm{p}-l o v G-6 \mathrm{xHis}-A D H 2 \mathrm{t}\end{array}$ & pSL05 from Xu, et al, 2013 \\
\hline $\mathrm{pCB} 21$ & $\begin{array}{l}2 \mu ; A m p R ; T R P 1 ; A D H 2 \mathrm{p}-6 \mathrm{xHis}-m / c G- \\
A D H 2 t ; A D H 2 \mathrm{p}-\text { lov } G \text {-6xHis- } A D H 2 \mathrm{t}\end{array}$ & This study \\
\hline pCB27 & $\begin{array}{l}2 \mu ; A m p R ; H I S 3 ; A D H 2 \mathrm{p}-6 \mathrm{xHis}-m / c G- \\
A D H 2 t ; A D H 2 \mathrm{p}-\text { lov } G-6 \mathrm{xHis}-A D H 2 \mathrm{t}\end{array}$ & This study \\
\hline pCB19 & $\begin{array}{l}2 \mu ; A m p R ; L E U 2 ; A D H 2 \mathrm{p}-\text { lovA-6xHis- } \\
A D H 2 \mathrm{i} \text {; } A \text { - } 2 \mathrm{p}-6 \mathrm{xHis}-\text { lovD9-ADH2t }\end{array}$ & This study \\
\hline
\end{tabular}

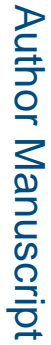

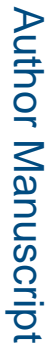

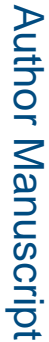


Table 3:

Plasmid copy number characterization. The percent of cells carrying plasmids was calculated by comparing the colony forming units (CFU) on the appropriate selective media plates to the CFU on complex media plates. For selection markers, pLovB carried ura3, pCB21 carried trp1, and pCB19 carried leu2. The copy number averaged over all cells was calculated by qPCR using the standard curve method, and the copy number per cell carrying plasmid was calculated by taking the average copy number for all the cells and dividing it by the fraction of cells carrying the plasmid of interest.

\begin{tabular}{|l|l|l|l|l|}
\hline & $\begin{array}{l}\text { Time post } \\
\text { inoculation }\end{array}$ & $\begin{array}{l}\text { Percent of } \\
\text { cells carrying } \\
\text { plasmid }\end{array}$ & $\begin{array}{l}\text { Copy number per } \\
\text { cell carrying } \\
\text { plasmid }\end{array}$ & $\begin{array}{l}\text { Copy number } \\
\text { averaged over all } \\
\text { cells }\end{array}$ \\
\hline \multirow{2}{*}{ pLovB } & 24 hours & $39.3 \pm 12.7$ & $18.4 \pm 5.7$ & $6.8 \pm 1.9$ \\
\cline { 2 - 5 } & 48 hours & $47.5 \pm 12.2$ & $22.8 \pm 3.3$ & $10.4 \pm 1.0$ \\
\cline { 2 - 5 } & 72 hours & $44.1 \pm 4.1$ & $22.5 \pm 5.3$ & $9.7 \pm 1.9$ \\
\hline \multirow{2}{*}{ pCB21 } & 24 hours & $40.7 \pm 13.5$ & $75.6 \pm 38.7$ & $25.6 \pm 8.4$ \\
\cline { 2 - 5 } & 48 hours & $43.3 \pm 11.0$ & $108.6 \pm 20.7$ & $45.1 \pm 7.7$ \\
\cline { 2 - 5 } & 72 hours & $37.4 \pm 5.0$ & $81.5 \pm 19.3$ & $30.0 \pm 5.5$ \\
\hline \multirow{2}{*}{ pCB19 } & 24 hours & $51.7 \pm 10.8$ & $18.1 \pm 3.1$ & $9.5 \pm 3.0$ \\
\cline { 2 - 5 } & 48 hours & $52.8 \pm 12.7$ & $25.2 \pm 7.5$ & $12.4 \pm 0.7$ \\
\cline { 2 - 5 } & 72 hours & $44.7 \pm 12.4$ & $28.6 \pm 6.8$ & $12.1 \pm 2.4$ \\
\hline
\end{tabular}

